\title{
ACOSO PSICOLÓGICO (MOBBING) EN EL ÁMBITO EDUCATIVO
}

\author{
Antonio Félix Raya Trenas \\ Javier Herruzo Cabrera \\ Ma José Pino Osuna
}

Departamento de Psicología. Universidad de Córdoba

\section{RESUMEN}

El presente estudio intenta llevar a cabo una medición de los niveles de acoso psicológico en una muestra de profesores de colegios de educación infantil y primana $e$ institutos de secundaria y describir las conductas de acoso más frecuentes entre estos 220 profesores (93 hombres y 127 mujeres) de la provincia de Córdoba. Se describen también las relaciones entre sexo, nivel educativo, situación contractual, localidad de trabajo, edad, antigüedad y acoso psicológico y las conductas de acoso más frecuentes. Los resultados muestran que cada sujeto sufre una media de más de cuatro conductas de acoso

\footnotetext{
Los autores del presente manuscrito pertenecen a la Universidad de Córdoba (Departamento de Psicología), como becarto FPI el primero y como profesores titulares de universidad los siguientes. Todos pertenecen al grupo de Investigación Comportamientos de Riesgo, Salud y Seguridad Laboral (código PAl: HUM-775), dirigido por el Dr. Javier Herruzo Cabrera, desde el que se desarrollan diversos proyectos de investigación. Uno de estos proyectos, que se desarrolla al amparo del programa de becas de formación de personal docente e investigador de la Junta de Andalucia (convocatoria 2003) es el denominado aMobbing en el profesorado andaluz», dentro del que se han llevado a cabo estudios como el descrito en estas páginas. Nota: Este estudio ha sido llevado a cabo en parte gracias al programa de becas de formación de personal docente e investigador de la Junta de Andalucía.

Correspondencia: Antonio Félix Raya Trenas. Dpto. de Psicología. Facultad de Ciencias de la Educación. C/San Alberto Magno s/n. 14071 Córdoba. E-mail: m02ratra@uco.es.
} 
psicológico de las sesenta descritas por el instrumento y que dos tercios de la muestra han indicado alguna de esas conductas. Por otro lado, ninguna de las variables demográficas contempladas resultaron ser determinantes con respecto al nivel de acoso psicológico. Respecto a las conductas de acoso, la comunicación y la reputación personal son los tipos de conductas más indicados. El análisis factorial describe dos factores principales referidos a reputación-aislamiento el primero y agresividad-tarea el segundo. Finalmente, se discute la semejanza entre estos factores y los de Leymann.

Palabras clave: PSICOLOGIA DEL TRABAJO, ACOSO PSICOLÓGICO, PROFESORES.

\section{SLIMMARY}

The present study tries to carry out a measurement of the mobbing level in a sample of nursery-primary education and high school teachers and it tries to describe the more frequent mobbing behaviours between these 220 teachers ( 93 male and 127 female) of the province of Cordoba. The relations between sex, educational level, contractual situation, locality of work, age, seniority and mobbing and the more frequent mobbing behaviours are also described. The results show that every subject undergoes an average of more than four mobbing behaviours of the sixty described by the instrument and that two thirds of the sample have indicated some of those behaviours. On the other hand, none of the contemplated demographic variables result to be determining factors with respect to the mobbing level. Regarding the mobbing behaviours, communication and personal reputation are the more indicated kind of behaviours. Factorial analysis describes two principal factors, referred to reputationisolation the first and aggressiveness-work the second one. Finally, similarity between these factors and Leymann's factors is discussed.

Keywords: WORK PSYCHOLOGY, MOBBING, TEACHERS. 


\section{INTRODUCCIÓN}

Desde que Konrand Lorenz, etólogo alemán, hablara por primera vez de mobbing en los años setenta para referirse a algunos comportamientos animales en los que individuos más débiles se aliaban para atacar a otro más fuerte, se ha ido forjando un campo de estudio de especial relevancia en la actualidad, ya que tanto la situación descrita por Lorenz como otras análogas se pueden observar con elevada frecuencia en la sociedad y más concretamente en los entornos laborales, que se caracterizan, entre otras cosas, por la precariedad y la competitividad. En este sentido, fue Leymann (1990) quien aplicó el término mobbing al entorno laboral, para definirlo como una forma de violencia psicológica sistemática sobre otra persona, durante un tiempo prolongado, en el lugar de trabajo. Por otro lado, en los últimos años han surgido otras definiciones análogas como bullying (violencia entre iguales) (Adams, 1992), harassement (hostigamiento) (Vartia, 1993) o acoso moral (Hirigoyen, 2001). El creciente interés por este fenómeno crea la necesidad de un término con identidad, para lo que se estima oportuno el empleo de la expresión acoso psicológico, aunque hay que ser conscientes de que el término mobbing está siendo usado con frecuencia en la actualidad y se puede esperar su pronta se castellanización, como ocurrió con la palabra estrés.

Aunque la literatura sobre el tema es escasa, se puede decir que el acoso psicológico está presente en todos los sectores laborales, aunque se ha detectado un mayor riesgo entre los docentes, los sanitarios y el personal de administraciones públicas o del estado (Fornes, 2003). Este es el motivo por el que la mayoría de los estudios se han realizado con personal sanitario y de la administración, aunque se encuentra un gran vacío en lo referente a la población docente.

Las primeras cifras con población general hablan de un porcentaje de afectados por acoso psicológico que va desde el 3,5\% descrito por Leymann (1992) y el 3\% obtenido por López y Camps del Saz (1997), pasando por el 8,6\% descrito por Einarsen y Skogstad (1996) hasta el $16 \%$ obtenido por Piñuel y Oñate (2002).

En un estudio llevado a cabo por González de Rivera y colaboradores con una muestra de 125 sujetos de ambos sexos de los cuales 29 
estaban diagnosticados de acoso psicológico, usando como instrumento una versión en fase de adaptación a España del LIPT (Leymann Inventory of Psychological Terrorization) original de Leymann de 45 items, que en esta versión pasa a tener 60 items, el LIPT-60, se concluyó lo siguiente: la media de conductas de acoso psicológico sufridas por las personas diagnosticadas fue de 29, con un IGAP (indice global de acoso psicológico calculado dividiendo la suma de las puntuaciones de cada item por el número de items) de 1,23. En cuanto a las personas de población diagnosticada, se obtuvo una media de 4 conductas de acoso psicológico sufridas, con un IGAP de 0,09 . Entre otros datos de interés también se concluyó que el $70 \%$ de la muestra manifestaba alguna conducta de acoso psicológico (González de Rivera y Rodríguez-Abuin, 2003). En otro estudio realizado en México con profesorado universitario y usando el mismo instrumento, se obtuvo un NEAP (número de estrategias de acoso psicológico) de 7 y un IGAP (índice global de acoso psicológico) de 0,19 (Pando, Aranda, Aldrete, Torres y Chavero, 2006).

En cuanto a las variables epidemiológicas que pueden determinar la mayor o menor exposición al acoso psicológico, uno de los aspectos más tenidos en cuenta es la influencia del género como posible variable. En este sentido, del análisis de los principales estudios realizados se puede extraer que en ningún caso se encontraron diferencias significativas entre ambos géneros (Einarsen y Skogstad, 1996; Fidalgo y Piñuel, 2004; González de Rivera y Rodríguez Abuin, 2003; Leymann, 1997), aunque en un estudio reciente realizado con personal de transportes se ha encontrado una mayor tasa de acoso psicológico en mujeres (Moreno, Rodríguez, Garrosa, Morante y Rodríguez, 2005).

Respecto a la edad, otra de las variables más estudiadas, existe cierto desacuerdo entre los principales autores, pues mientras que unos afirman que no existen diferencias significativas (Leymann, 1997), otros sí que encuentran diferencias, señalando mayor incidencia del acoso psicológico en jóvenes (Einarsen y Raknes, 1997; Einarsen y Skogstad, 1996; Piñuel y Oñate, 2002) o por el contrario, mayor incidencia en mayores (González de Rivera y Rodríguez Abuin, 2003).

En cuanto a la antigüedad, también se encuentran datos contrapuestos, pues mientras que algunos autores afirman que el 
acoso psicológico se da más en los trabajadores eventuales (Piñuel y Oñate, 2002), otros señalan una correlación positiva entre el acoso psicológico y la antigüedad (González de Rivera y Rodríguez Abuin, 2003).

Por otro lado, muchos esfuerzos se han dirigido a tratar de aislar aquellas conductas que se consideran más frecuentes en las distintas situaciones de acoso psicológico. En este sentido, Knorz y Zapf (1996) publicaron una lista de comportamientos de acoso psicológico identificados en el Sur de Alemania a través de entrevistas cualitativas. Posteriormente, Leymann (1997) señaló 5 como las principales conductas de acoso psicológico, que se dividen a su vez en otras, hasta un total de 45 conductas de acoso que componen el LIPT (Leymann Inventory of Psychological Terrorization). Estas 5 categorías son:

- Las que afectan a las posibilidades de que la víctima se comunique adecuadamente.

- Las que afectan a las posibilidades de la víctima para mantener contacto social.

- Las que afectan a las posibilidades de la víctima para mantener su reputación personal.

- Las que afectan a la situación laboral de la víctima.

- Las que afectan a la salud física de la víctima.

En España, Piñuel y Oñate (2002) elaboraron un estudio denominado "Barómetro Cisneros II sobre violencia en el entorno laboral». Como resultado de esta encuesta, que fue aplicada en la zona centro del país sobre todos los sectores laborales, elaboraron una lista de comportamientos de acoso psicológico más frecuentes. También González de Rivera (2003) elaboró una lista de las 10 conductas más frecuentes, extraídas de la aplicación del LIPT-60, la versión adaptada a nuestro país del LIPT original de Leymann. Estas 10 conductas más frecuentes son:

- Hablar mal de alguien a sus espaldas.

- Miradas y gestos despectivos.

- Rechazo de contacto por alusiones o gestos.

- Tratarle como si fuera transparente o invisible.

- Evaluaciones de rendimiento malas o hirientes.

- Difundir rumores. 
- Críticas continuas sobre el trabajo de alguien.

- Limitaciones de sus posibilidades de expresión por los jefes.

- Cuestionar todas sus decisiones.

- Asignarle tareas muy por debajo de su categoría o capacitación.

A pesar de los estudios mencionados, la mayoría de los datos que actualmente se manejan son el resultado de apreciaciones cualitativas junto con análisis teóricos, casi nunca basados en estudios empíricos sistemáticos.

Junto a la necesidad de unos datos epidemiológicos resultantes de instrumentos y estudios fiables, también resulta interesante extraer cierta información relativa a algunas variables demográlicas o características personales presentes en episodios de acoso psicológico y en sus protagonistas. Para ello nos proponemos los siguientes objetivos:

- Llevar a cabo una medición de los niveles de acoso psicológico en una muestra de profesores de Educación Infantil, Primaria y Secundaria.

- Estudiar la relación de determinadas variables como la edad, el sexo, la situación contractual del trabajador, la localización del centro o el nivel de estudios que se imparte, con las posibilidades de sufrir acoso psicológico por parte de otras personas del entorno de trabajo.

- Describir las conductas de acoso psicológico más frecuentes entre la población docente de la provincia de Córdoba.

- Extraer factores a partír de esas conductas que nos permitan comparar distintas categorías de acoso psicológico y su incidencia en función de las distintas variables demográficas.

\section{MÉTODO}

\section{Sujetos}

La muestra estaba compuesta por 220 profesores de la provincia de Córdoba, seleccionados al azar, aunque se tuvo en cuenta que los sujetos seleccionados fueran representativos de la población docente de la provincia en cuanto a las variables demográficas tenidas en cuenta en este estudio, distribuyéndose de la siguiente forma: respecto al nivel educativo que se impartía, se distinguió entre centros de 
Educación Infantil y Primaria $(n=136)$ y centros de Secundaria $(n=84)$; en cuanto a la población en que estaba situado el centro, se distinguió entre la capital $(n=95)$ y las poblaciones de la provincia $(n=125)$; también se tuvo en cuenta la situación contractual, distinguiendo entre funcionarios $(n=172)$ e interinos $(n=45)$ (tres no contestaron); por último, en función del sexo, la muestra quedó distribuida en 93 hombres y 127 mujeres.

La media de edad de la muestra era $42,26(S=9,81)$ y la media de antigüedad en la profesión 17,04 $(S=10,30)$. Respecto a estas dos últimas variables, la muestra cumplía la condición de normalidad según la prueba de Kolmogorov - Smirnov, pues no se hallaron diferencias significativas respecto a la curva normal, siendo $Z=0,933$ ( $p=$ $0,349>0,05)$ para la edad y $Z=1,303(p=0,067>0,05)$ para la antigüedad.

\section{Instrumentos}

Para la recogida de la información se elaboró un cuestionario que garantizaba la confidencialidad de los datos recogidos, ya que se preservó el anonimato de los participantes. El instrumento se componía de las siguientes partes:

- Cuestionario elaborado para este estudio sobre datos demográficos como el sexo, la edad, la antigüedad, la situación contractual, la localidad en que se halla el centro o el nivel educativo impartido.

- Cuestionario de Estrategias de Acoso Psicológico (LIPT - 60) de González de Rivera y Rodríguez-Abuin (2003) que es un cuestionario escalar autoadministrado que objetiva y valora 60 estrategias de acoso psicológico, derivado del LIPT original dicotómico de 45 items de Leymann. En este cuestionario, el sujeto debía señalar con una puntuación entre 0 y 4 la intensidad con que experimentaba las situaciones descritas en los distintos items.

\section{Procedimiento}

Tras seleccionar la muestra, se tomó contacto con los centros educativos cuyo profesorado iba a participar en el estudio. Para 
acceder a este profesorado y hacerle llegar el instrumento se recurría a alguna persona del equipo directivo (director, jefe de estudios...) a quien se le exponía la finalidad del proyecto y la manera en que se iba a llevar a cabo.

Los cuestionarios fueron cumplimentados en la primavera de 2004 por los profesores y profesoras que accedian a ello de manera voluntaria. Este profesorado pertenecía a centros de toda la provincia de Córdoba seleccionados en función de su localización y del nivel educativo que en ellos se impartía.

Una vez recogidos los cuestionarios se pasó al vaciado de los datos y su posterior análisis mediante el programa estadístico SPSS. Dicho análisis consistió en una descripción de los niveles medios de acoso psicológico manifestados por la muestra, para pasar posteriormente a comparar la puntuación media en mobbing obtenida por la muestra en función de las variables demográficas contempladas en el estudio, para lo que se llevaron a cabo diversas pruebas $T$ y algunas correlaciones de Pearson. Posteriormente se seleccionaron las 10 conductas de acoso más señaladas por la muestra y se llevó a cabo un análisis factorial para tratar de aislar categorías concretas de conductas de acoso psicológico y sus diferencias entre los distintos grupos de la muestra.

En este estudio descriptivo se han tenido en cuenta las siguientes variables: número de estrategias de acoso psicológico (NEAP), índice global de acoso psicológico (IGAP), sexo, edad, antigüedad en la profesión, situación contractual, población en que se encuentra el centro educativo y nivel educativo que se imparte.

\section{RESULTADOS}

El primero de los objetivos que nos habíamos propuesto era llevar a cabo una medición de los niveles de acoso psicológico en la muestra estudiada. Para el análisis de estos niveles de acoso psicológico encontrados se hizo referencia a dos formas de medida que nos ofrecian información sobre la cantidad de estrategias de acoso psicológico sufridas la primera y sobre la intensidad con que se daban dichas situaciones la segunda.

La primera de estas formas de medida es la que llamábamos NEAP, que consiste en el recuento simple de todas las respuestas distintas 
de cero o número de estrategias de acoso psicológico que manifiesta haber sufrido cada sujeto. La media obtenida en esta muestra fue de $4,49(S=8,41)$ aunque existía gran diferencia entre algunos sujetos, oscilando entre 0 y 56 como puede observarse en la figura 1, de ahí la elevada desviación típica resultante.

Dividiéndolo por rangos, haciendo grupos en función del número de estrategias manifestadas, se obtuvo que $165(75 \%)$ manifestaban menos de 5 estrategias acoso psicológico, 22 (10\%) manifestaban entre 5 y $9,16(7,3 \%)$ entre 10 y $14,6(2,7 \%)$ entre 15 y 19 y $11(5 \%)$ más de 20 . Además, $147(66,8 \%)$ personas de las que componian la muestra señalaron al menos una de las situaciones descritas en el cuestionario.

Respecto a la otra manera de medir el mobbing a la que llamamos IGAP (índice global obtenido sumando los valores asignados a cada estrategia de acoso y dividiendo por el número total de estrategias consideradas, que son 60 ), la media de la muestra fue de 0,14 ( $S=$ $0,37)$, aunque hay que tener en cuenta que $73(33,2 \%)$ sujetos puntuaron 0 y que existian también sujetos con puntuaciones bastante elevadas, siendo la máxima de 3,53, como puede verse en la figura 2 .

Figura 1. Porcentaje de sujetos según su puntuación en NEAP

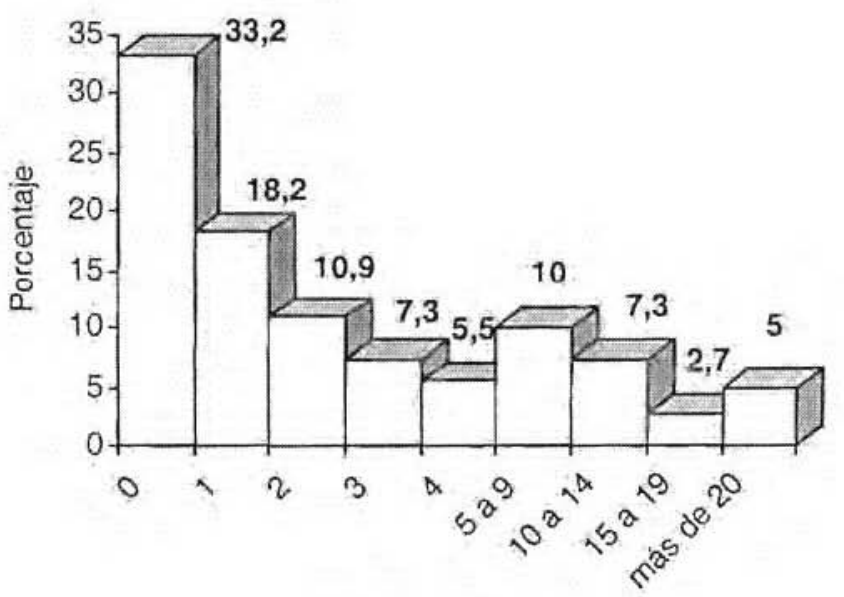

Pưntuación en NEAP 
Figura 2. Porcentaje de sujetos según su puntuación en IGAP

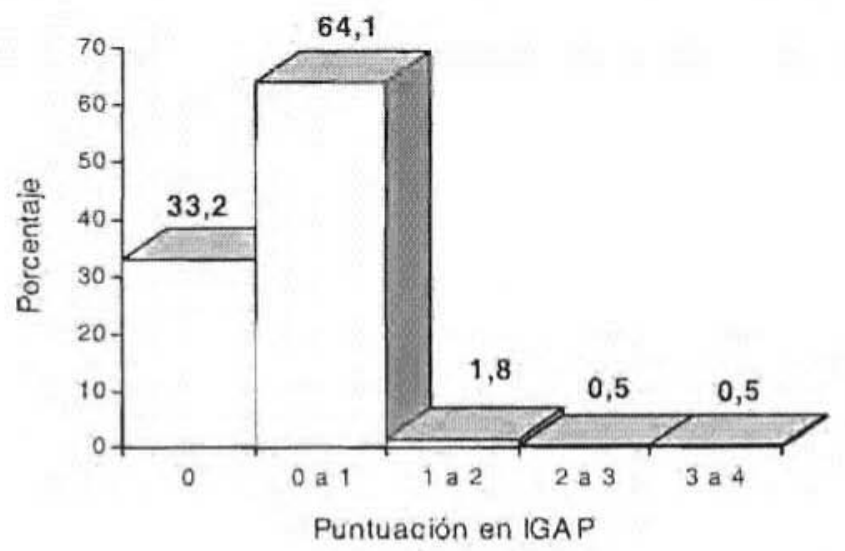

Otro de los objetivos planteado en este estudio era analizar la influencia de factores demográficos como el sexo, la situación contractual, la edad, la antigüedad, el nivel docente o la población de trabajo en los niveles de acoso psicológico. Respecto a este objetivo, hemos obtenido los siguientes datos:

En lo referente a la variable "sexo", las puntuaciones obtenidas en las distintas mediciones de las estrategias de acoso psicológico fueron algo distintas, obteniendo las siguientes medias: NEAP (hombres) $=4,89(\mathrm{~S}=8,89)$, NEAP (mujeres) $=4,19(\mathrm{~S}=8,06)$, IGAP (hombres) $=0,13(\mathrm{~S}=0,29)$, IGAP (mujeres) $=0,14(\mathrm{~S}=0,42)$.

Al aplicar la prueba $T$ de Student para comprobar si la diferencia de medias entre sexos era significativa, se obtuvo una $t=0,612(p=$ $0,541>0,05$ ) para las medias de NEAP según sexos y una $t=-0,153$ $(p=0,878>0,05)$ para las medias de IGAP según sexos, por lo que podemos decir que la diferencia entre ambos sexos no era significativa.

Respecto a la "situación contractual», el análisis en función de esta variable arrojó datos parecidos a los obtenidos respecto a la variable sexo, pues las medias de las puntuaciones obtenidas fueron las siguientes: $\operatorname{NEAP}$ (funcionarios) $=4,31(\mathrm{~S}=8,33), \operatorname{NEAP}$ (interinos) $=5,31 \quad(\mathrm{~S}=8,98)$, $\operatorname{IGAP}$ (funcionarios) $=0,14(\mathrm{~S}=0,40), \operatorname{IGAP}$ (interinos) $=0,12(\mathrm{~S}=0,22)$.

La prueba T de Student arrojó una $t=-0,707(p=0,480>0,05)$ para las medias de NEAP según situación laboral y una $t=0,317(p=0,752>0,05)$ 
para las medias de IGAP según situación laboral, por lo que podemos decir que tampoco existían diferencias significativas entre funcionarios e interinos.

Respecto a la variable «población en que se encuentra el centro educativo", las medias obtenidas en NEAP e IGAP no fueron muy diferentes en función del lugar en que se encontraba el centro. Así, para los maestros de la capital, las medias de NEAP e IGAP fueron $4,63(S=7,49)$ y $0,12(S=0,20)$ respectivamente; para los maestros de centros situados en la provincia, la media de NEAP fue de 4,38 $(S=9,07)$ y la de IGAP fue $0,15(S=0,46)$.

La prueba T de Student tampoco indicó la existencia de diferencias significativas entre las medias de NEAP e IGAP de docentes de la capital y de la provincia, ya que se obtuvo una $t=0,223(p=0,824>0,05)$ para NEAP y $t=-0,746(p=0,456>0,05)$ para IGAP.

Por otro lado, en lo referente a la variable nivel docente, los resultados obtenidos no distaban demasiado de los de la muestra general, existiendo alguna pequeña diferencia. Las medias de NEAP e IGAP para el profesorado de Educación Infantil y Primaria fueron $3,83(S=7,07)$ y $0,11(S=0,29)$ respectivamente. Para el profesorado de Educación Secundaria, estas medias fueron $5,55(S=10,17)$ para NEAP y $0,17(S=0,47)$ para IGAP.

La prueba T de Student dio como resultado $t=1,475(p=0,142>0,05)$ para NEAP y $t=1,120(p=0,246>0,05)$ para IGAP, luego no se pudo decir que existieran diferencias significativas entre profesores de Educación Infantil/Primaria y Secundaria respecto al acoso psicológico.

Por último, para analizar la relación entre las variables NEAP e IGAP y las variables numéricas edad y antigüedad, se calculó el coeficiente de correlación de Pearson, que en ningún caso resultó ser significativo, como se muestra en la tabla 1.

Tabla 1. Correlaciones de Pearson entre el NEAP y el IGAP y las variables edad y antigüedad

\begin{tabular}{|l|c|c|}
\cline { 2 - 3 } \multicolumn{1}{c|}{} & EDAD & ANTIGÜEDAD \\
\hline NEAP & $r=0,025(p=0,709>0,05)$ & $r=0,037(p=0,599>0,05)$ \\
\hline IGAP & $r=0,089(p=0,190>0,05)$ & $r=0,118(p=0,092>0,05)$ \\
\hline
\end{tabular}


Respecto a las conductas de acoso psicológico más frecuentes que se pueden encontrar en la muestra estudiada, se llevó a cabo un recuento de los sujetos que habían señalado cada uno de los distintos items del cuestionario. De este recuento, se obtuvo como resultado que las conductas de acoso psicológico más frecuentes son las que se enumeran en la tabla 2, junto al porcentaje de sujetos que las han señalado.

Tabla 2. Principales conductas de acoso psicológico señaladas por la muestra

\begin{tabular}{|l|c|}
\hline \multicolumn{1}{|c|}{ CONDUCTAS DE ACOSO PSICOLÓGICO SEÑALADAS } & $\%$ total \\
\hline 2. Le interrumpen cuando habla. & 36,8 \\
\hline 5. Critican su trabajo. & 30,5 \\
\hline 1. Sus superiores no le dejan expresarse o decir lo que tienen que decir. & 28,2 \\
\hline 3. Sus compañeros le ponen pegas para expresarse o no le dejan hablar. & 20,9 \\
\hline 29. Sus decisiones son cuestionadas o contrariadas. & 16,4 \\
\hline 58. Controlan de manera muy estricta su horario. & 15,5 \\
\hline 11. Ignoran su presencia, no responden a sus preguntas & 15,0 \\
\hline 10. No le miran o le miran con desprecio o rechazo. & 12,7 \\
\hline 4. Le gritan o le regañan en voz alta. & 11,9 \\
\hline 36. Le sobrecargan sin cesar con tareas diferentes. & 11,9 \\
\hline
\end{tabular}

Por otro lado, para tratar de obtener una información más global sobre el tipo de conductas de acoso psicológico más frecuentes en la muestra en función de las variables demográficas, se llevó a cabo un análisis factorial mediante el que se pretendía aislar factores que determinaran distintas formas de acoso psicológico que pudieran variar entre las distintas submuestras.

El primer paso para llevar a cabo este tipo de análisis consistió en la extracción y análisis de los componentes principales, de modo que el factor que representara mayor variabilidad pasara a ser el primer componente principal y así sucesivamente, con el objetivo de obtener un conjunto de nuevas variables no correlacionadas entre sí. 
Partiendo de la regla de conservar aquellos factores cuyos autovalores fueran mayores que 1 (González López, 2003), se obtuvieron ocho factores, que explicaban el $83,09 \%$ de la varianza. Estos factores quedan representados en la tabla 3.

Tabla 3. Porcentaje de la varianza total explicada por cada factor resultante del análisis factorial

$\begin{array}{ccccc}\text { Factor } & \text { Varianza explicada } & & \text { \% de la varianza } & \text { \% acumulado } \\ \mathbf{1} & 20,206 & & 33,677 & 33,677 \\ \mathbf{2} & 18,526 & & 30,877 & 64,554 \\ \mathbf{3} & 2,671 & 4,451 & 69.006 \\ 4 & 1,902 & 3,170 & 72,176 \\ \mathbf{5} & 1,701 & 2,834 & 75,010 \\ \mathbf{6} & 1,666 & 2,776 & 77,786 \\ \mathbf{7} & 1,626 & 2,710 & 80,496 \\ \mathbf{8} & 1,556 & 2,549 & 83,090\end{array}$

Siguiendo a García, Gil y Rodríguez (2000) que aconsejaban un mínimo de tres variables o ítems en cada factor, sólo 4 de los factores obtenidos ( 1 , 2, 3 y 7$)$ cumplían esta condición. Además, los factores 3 y 7 hacían referencia a un contenido menos general en comparación con los factores 1 y 2, por lo que se decidió estudiar sólo estos dos factores que en conjunto explicaban el $64,554 \%$ de la varianza total, que era una cifra bastante considerable.

Para tratar de poner un nombre a cada factor se analizó el contenido de los ítems que componían cada uno, obteniendo lo siguiente:

- Factor 1: reputación-aislamiento. Los ítems que componían este factor, que eran 36 , hacían referencia a conductas como las críticas continuas, ridiculización, falta de comunicación o aislamiento.

- Factor 2: agresividad-tarea. Los 30 ítems que lo componían en este caso hacian referencia a conductas relativas a agresiones físicas y verbales y a tareas inadecuadas o peligrosas.

Una vez aislados los dos principales factores que se obtuvieron mediante el análisis factorial, se halló el valor de IGAP en esta muestra para cada uno de los factores, obteniéndose un IGAP medio de 0,16 $(S=0,46)$ para el factor denominado reputación-aislamiento y un IGAP medio de $0,06(S=0,30)$ para el factor denominado agresividad-tarea. Además, 127 sujetos obtuvieron valores distintos de cero en el primer factor, mientras que 59 lo hicieron en el segundo. 
En cuanto a las variables demográficas, se compararon las medias obtenidas en cada factor por las distintas submuestras para tratar de describir las posibles diferencias existentes. Esta comparación de medias queda representada en la tabla 4.

Tabla 4. Comparación de medias obtenidas por cada submuestra en el IGAP de cada factor mediante prueba T de Student

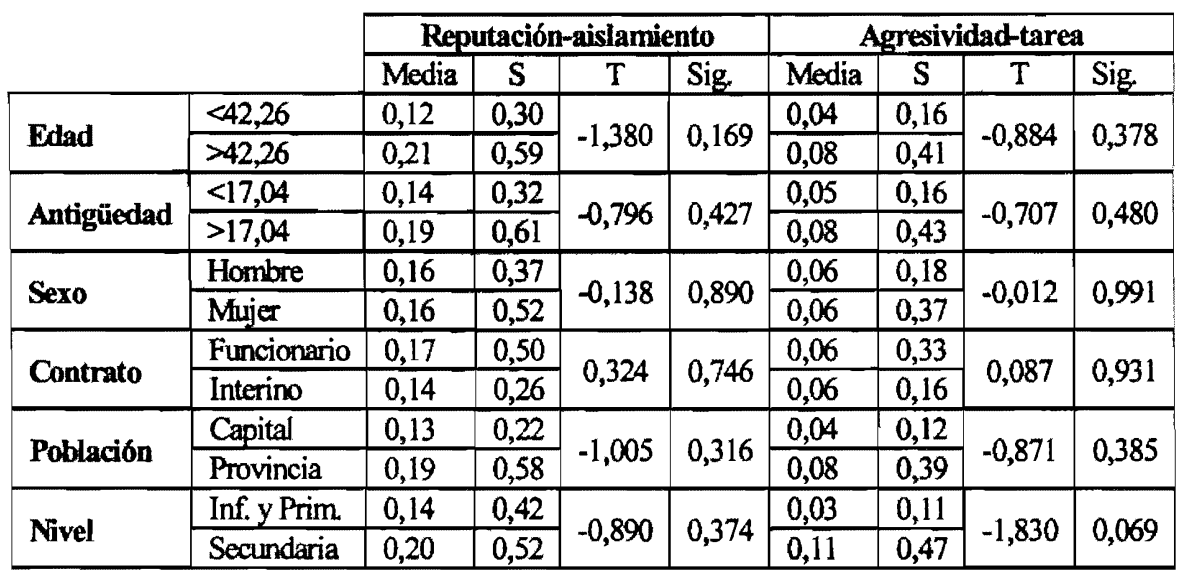

Mediante los datos obtenidos en las distintas pruebas para la igualdad de medias, se comprobó en todos los grupos formados en la muestra que el factor reputación-aislamiento obtuvo puntuaciones mayores que el factor agresividad-tarea en todos los casos. En función de las variables demográficas se observaron algunas diferencias interesantes como las presentadas por el factor reputaciónaislamiento en función de la edad por un lado o las diferencias presentadas por el factor agresividad-tarea en función del nivel docente por otro. No obstante, la elevada dispersión de la muestra no permitía hablar de diferencias significativas en ningún caso.

\section{DISCUSIÓN}

Antes de pasar a comentar los resultados obtenidos en el estudio, resulta necesario destacar que el instrumento utilizado consistía en un autoinforme y que por lo tanto, la información obtenida depende de las estimaciones subjetivas de los propios sujetos, a pesar de lo cual se pueden extraer algunas conclusiones interesantes. 
A la vista de la distribución de la población respecto a la variable NEAP e IGAP, se puede decir que el acoso psicológico es un fenómeno que afecta en niveles bajos a la población general, existiendo incluso un buen porcentaje de sujetos que no lo padecen en absoluto. Igualmente existe una minoría que ha obtenido puntuaciones bastante altas, por lo que se puede afirmar que el acoso psicológico es un fenómeno bastante frecuente en la población, aunque sólo alcanza niveles excesivos en una pequeña parte.

En la medición de los niveles de acoso psicológico a través de las dos formas establecidas, se han obtenido puntuaciones medias algo más elevadas que las obtenidas por González de Rivera y RodríguezAbuin (2003) en su aplicación experimental con población normal del LIPT-60, instrumento usado en esta investigación. Las puntuaciones obtenidas en este estudio fueron de 4 para NEAP y 0,09 para IGAP. En nuestro estudio se han obtenido medias de 4,49 para NEAP y 0,14 para IGAP. Estos resultados se pueden considerar similares, teniendo en cuenta que no se han excluido a los sujetos clínicamente significativos. No obstante, estas puntuaciones son inferiores a las obtenidas por Pando y cols. (2006) con población universitaria mexicana, usando el mismo instrumento, pues obtuvieron un NEAP de 7 y un IGAP de 0,19.

Se puede decir que cada docente sufre de 4 a 5 estrategias de acoso psicológico como término medio y que, al igual que señalaba González de Rivera y Rodríguez-Abuin (2003) en su estudio, dos terceras partes de la población han manifestado alguna de las estrategias de acoso señaladas en el cuestionario.

Al analizar las distintas variables demográficas implicadas en el estudio como son el sexo, la situación laboral, la edad, la antigüedad en el puesto, el nivel docente o la localidad de trabajo, se han obtenido resultados en parte similares a los esperados, aunque con diferencias importantes.

Por un lado, al igual que señalaban Einarsen y Skogstad (1996), Fidalgo y Piñuel (2004), González de Rivera y Rodríguez-Abuin (2003), Leymann (1997) y Piñuel y Oñate (2002), no se obtuvieron diferencias significativas respecto al sexo, encontrando incongruencias con los datos obtenidos por Moreno y cols. (2005) con personal de transportes. No obstante, el hecho de que en la profesión docente exista un mayor número de mujeres que de hombres, al contrario que en el sector 
de los transportes, puede jugar un papel importante en cuanto al componente discriminatorio del acoso psicológico.

Algo similar ocurre con la variable situación laboral, en la que, a pesar de existir diferencias algo superiores, no se pudieron considerar significativas como indican los resultados. Respecto a esta variable, los datos contradicen a las afirmaciones hechas por Piñuel y Oñate (2002) que sí apreciaban ciertas diferencias en las puntuaciones de trabajadores fijos y eventuales. Estos resultados pueden deberse a que el profesor interino no es un trabajador eventual normal, pues no está sometido a las presiones y controles que pueden sufrir otro tipo de trabajadores eventuales. El profesor interino normalmente suele implicarse poco en las luchas de poder de la organización.

Podemos decir que la edad y la antigüedad tampoco son variables que influyan en la posibilidad de sufrir acoso psicológico ni en la intensidad de éste, como señalaba Leymann (1997). En este caso, también hay que señalar diferencias respecto a los datos señalados por autores como Einarsen y Raknes (1997), Einarsen y Skogstad (1996), Fornés (2003), González de Rivera y Rodríguez-Abuin (2003) y Piñuel y Oñate (2002), cuyos estudios daban como resultado una mayor incidencia del acoso psicológico en jóvenes y trabajadores eventuales.

Las dos variables medidas en este estudio y que nunca fueron tenidas en cuenta en otro estudio sobre acoso psicológico como son el nivel educativo o la población en que se encuentra el centro, no han resultado ser variables determinantes. Por ello, podemos decir que prácticamente ninguno de los factores demográficos estudiados puede ser considerado un factor determinante ante el acoso psicológico.

Respecto a las conductas de acoso más frecuentes, si atendemos a la clasificación que estableció Leymann (1997), se puede afirmar que los comportamientos de acoso más frecuentes entre el profesorado son los que afectan a las posibilidades de que la víctima se comunique adecuadamente (4 de las 10 conductas más frecuentes) y los que afectan a las posibilidades de la víctima para mantener su reputación personal ( 3 de las 10 conductas más frecuentes). El resto hace referencia a la situación laboral de la víctima ( 2 conductas) y a las posibilidades de la víctima para mantener contacto social (1 conducta), no encontrando entre las más frecuentes ninguna conducta referente a la salud física de la víctima. 
En lo referente a los dos factores obtenidos a raíz de este estudio, se puede decir que en el ámbito docente se da una mayor concentración de las conductas de acoso psicológico, de manera que quedan divididas en dos grandes grupos: por un lado el factor reputación-aislamiento que se puede decir que aglutina a tres de las categorias establecidas por Leymann (1997) como eran las que afectan a la comunicación, las que afectan al contacto social y las que afectan a la reputación personal, y por otro lado el factor agresividad-tarea que aglutina a las conductas que afectan a la situación laboral y las que afectan a la salud física de la víctima.

De cara al futuro, se estiman necesarios estudios con muestras más amplias, tanto de personal docente como de otros sectores laborales, sobre todo en lo que a personas afectadas por el acoso psicológico se refiere, para poder obtener datos más fiables. Por otro lado, también se pueden establecer nuevas distinciones dentro del personal docente como la pertenencia a centro público o privado 0 considerar también variables de tipo clínico que nos puedan llevar a descubrir los efectos del acoso psicológico.

\section{REFERENCIAS BIBLIOGRÁFICAS}

Adams, A. (1992). Bullying at work: how to confront and overcome it. London: Virago Press.

Einarsen, S. \& Raknes, B. I. (1997). Harassment at work and the victimization of men. Violence and Victims, 12, 247-263.

Einarsen, S. \& Skogstad, A. (1996). Bullying al work: Epidemiological findings in public and private organizations. European Journal of Work and Organizational Psychology, 5, 185-201.

Fidalgo, A. M. y Piñuel, I. (2004). La escala Cisneros como herramienta de valoración del mobbing. Psicothema, 16(4), 615-624.

Fornés, J. (2003, Febrero). Acoso psicológico en el mundo laboral.

Responsabilidades y control. Congreso Virtual Interpsiquis 2003. García, E., Gil, J., y Rodríguez, G. (2000). Análisis factorial. Madrid: La Muralla / Salamanca: Hespérides.

González de Rivera, J. L. (2003). El maltrato psicológico. Como defenderse del mobbing y otras formas de acoso. Madrid: Espasa. 
González de Rivera, J. L. y Rodríguez-Abuin, M. (2003). Cuestionario de estrategias de acoso psicológico: EI LIPT-60 (Leymann Inventory of Psychological Terrorization) en versión española. Psiquis, 24(2), 59-66.

González López, I. (2003). Determinación de los elementos que condicionan la calidad de la universidad: aplicación práctica de un análisis factorial. RELIEVE, $9(1)$, 83-96.

Hirigoyen, M. F. (2001). El acoso moral en el trabajo. Barcelona: Paidós.

Knorz, C. y Zapf, D. (1996). Mobbing. Eine extreme form sozialer Stresoren am Arbeitsplatz. Zeitschrift für Arbeits und Organisations Psychologie, 40, 12-21.

Leymann, $H_{:}$(1990). Mobbing and psychological terror at workplaces. Violence and victims, 5, 119-126.

Leymann, H. (1992). From bullying to expulsion from working life. Estocolmo: Pública.

Leymann, H. (1997). The mobbing encyclopaedia. http:// www.leymann.se

López, J. A. y Camps del Saz, P. (1997). Mobbing en puestos de trabajo de tipo administrativo. Medicina del trabajo, 6, (41-47).

Moreno, B., Rodríguez, A., Garrosa, E., Morante, M. E. y Rodríguez, R. (2005). Diferencias de género en el acoso psicológico en el trabajo: un estudio en población española. Psicologia em Estudo, Maringá, 10, 3-10

Pando, M., Aranda, C., Aldrete, M. G., Torres, T. M. y Chavero, O. (2006). Factores psicosociales de la organizacion asociados a la presencia de mobbing en docentes universitarios. Revista de Psiquiatria de la Facultad de Medicina de Barcelona. Vol 33, 42-47. Piñuel, I. y Oñate, A. (2002). La incidencia del mobbing 0 acoso psicológico en el trabajo en España. Resultados del Barómetro Cisneros II sobre violencia en el entorno laboral. Lan Harremanak, $7(2)(35-62)$.

Vartia, M. (1993). Psychological harassment (bullying, mobbing) at work. In K. Kauppinnen-Toropainen (Ed.) OECD panel group of women, work and health (pp 149-152). Helsinki: Ministry of Social Affairs and Health. 